\section{Control of Systems With Uncertain Initial Conditions}

Mazen Farhood and Geir E. Dullerud

\begin{abstract}
This note deals with the control of linear discrete-time systems with uncertain initial conditions. Specifically, we consider the problem where the initial condition is known to reside in a norm ball of some radius, and the input disturbance is constrained to satisfy an independent norm condition. The paper focuses on eventually periodic systems; these include both finite horizon and periodic systems as special cases. The main theorem provides exact synthesis conditions for the existence of eventually periodic controllers which both stabilize and provide performance in closed-loop control systems. These conditions are given in terms of a finite-dimensional semidefinite programming problem. We also give a version of the main result for the special case of linear time-invariant systems with uncertain initial states, and conclude with an illustrative example.
\end{abstract}

Index Terms-Eventually periodic systems, linear matrix inequalities (LMIs), orbits, time-varying systems, uncertain initial conditions.

\section{INTRODUCTION}

This note focuses on the control of linear discrete-time models with uncertain initial conditions, where the constraints placed on both the initial state and input disturbance are independent. The theory developed applies for linear time-varying (LTV) systems in general; however, as we seek finite dimensional synthesis results, we will focus our efforts on a special class of LTV systems, namely that of eventually periodic systems, and in this respect, continue the work started in [1]-[3]. Eventually periodic systems are aperiodic for an initial amount of time, and then become periodic afterwards. They contain both finite horizon and periodic systems as special cases, and naturally arise when controlling nonlinear systems along prespecified trajectories, notably those which eventually settle down into periodic orbits and those with uncertain initial states. We note that the results herein are novel even in the special case of linear time-invariant (LTI) systems.

Specifically, given an eventually periodic discrete-time system with an uncertain initial condition $x_{0}$, we derive necessary and sufficient conditions for the existence of an eventually periodic feedback controller which achieves closed-loop stability as well as the performance inequality (given in terms of 2-norms)

$$
\sup _{\left\|x_{0}\right\|+\|w\| \neq 0} \frac{\|z\|}{\max \left(\left\|x_{0}\right\|,\|w\|\right)}<\gamma,
$$

for some $\gamma$, where $w$ and $z$ denote the exogenous disturbances and errors respectively. It can be easily verified that

$$
\sup _{\left\|x_{0}\right\|+\|w\| \neq 0} \frac{\|z\|}{\max \left(\left\|x_{0}\right\|,\|w\|\right)}=\sup _{\left\|x_{0}\right\| \leq 1,\|w\| \leq 1}\|z\| .
$$

Manuscript received May 26, 2006; revised July 18, 2007 and January 09, 2008. Current version published December 10, 2008. This work was supported by National Science Foundation (NSF) Grant ITR-0085917 and AFOSR MURI Grant F49620-02-1-0325. Recommended by Associate Editor G. Chesi.

M. Farhood is with the Department of Aerospace and Ocean Engineering, Virginia Polytechnic Institute and State University (Virginia Tech), Blacksburg, VA 24061 USA (e-mail: farhood@vt.edu).

G. Dullerud is with the Department of Mechanical Science and Engineering, University of Illinois, Urbana, IL 61801 USA (e-mail: dullerud@illinois.edu).

Color versions of one or more of the figures in this paper are available online at http://ieeexplore.iee.org.

Digital Object Identifier 10.1109/TAC.2008.2007516
Observe that the norm constraints placed on $x_{0}$ and $w$ are independent. The synthesis conditions are provided in terms of a finite dimensional semidefinite programming problem; some seminal references on semidefinite programming are [4], [5] and the comprehensive book [6] which deals with various classes of convex optimization problems. We also specialize the main result to the case of LTI systems with uncertain initial states, and give an illustrative example.

The results here have potential uses in control problems involving switched and hybrid systems [7], [8]. For instance, as proposed in [9], one way to go about online path planning is to design a library of pre-specified maneuvers and then appropriately switch between these maneuvers online to generate the desired trajectory. Linearizing the nonlinear system equations about these maneuvers results in a family of finite horizon models composing a hybrid system. When the number of constituent models is very large, it may be very advantageous from a computational perspective to be able to design controllers for the constituent models separately while still managing to ensure stability and adequate performance. As we will not have in general accurate trajectory tracking due to model uncertainties and disturbances, it could prove very beneficial to design such controllers for plants with uncertain initial conditions using the results of this paper.

The general machinery used to obtain the results of this note is motivated by the work in [10]-[13], combined with the time-varying system machinery developed in [14], [15]. The literature in the area of timevarying systems is vast, and we refer the reader to [16] for a comprehensive list of general references. The LTV systems considered are allowed to have time-varying state-space dimensions. This is useful as it will be convenient for properly formulating the problem to have the exogenous disturbances vary in size over time. In addition, though it may seem a bit unconventional, a time-varying state dimension may naturally arise, for instance, when computing a minimal realization [17], [18] or deriving a reduced model [19], [20].

\section{PRELIMINARIES}

The set of real $n_{0} \times m_{0}$ matrices is denoted by $\mathbb{R}^{n_{0} \times m_{0}}$. If $S_{i}$ is a sequence of matrices, then $\operatorname{diag}\left(S_{i}\right)$ denotes their block-diagonal augmentation. The adjoint of an operator $X$ is written $X^{*}$, and we use $X \prec 0$ to mean it is negative definite. Given a bounded sequence of positive integers $n_{k}$ for $k=0,1,2, \ldots$, we define the normed space $\ell_{2}\left(\mathbb{R}^{n_{0}}, \mathbb{R}^{n_{1}}, \mathbb{R}^{n_{2}}, \ldots\right)$ to consist of elements $x=\left(x_{0}, x_{1}, x_{2}, \ldots\right)$, with each $x_{k} \in \mathbb{R}^{n_{k}}$, having a finite 2-norm $\|x\|$ defined by $\|x\|^{2}=\sum_{k=0}^{\infty}\left\|x_{k}\right\|^{2}<\infty$, where $\left\|x_{k}\right\|^{2}=x_{k}^{*} x_{k}$. The notation $\ell_{2}\left(\mathbb{R}^{n_{0}}, \mathbb{R}^{n_{1}}, \ldots\right)$ is abbreviated to $\ell_{2}$ when the dimensions are not relevant to the discussion. We use the notation $\|P\|_{\ell_{2} \rightarrow \ell_{2}}$ to denote the $\ell_{2}$-induced norm of a bounded linear mapping $P$ on $\ell_{2}$. Given $x \in \ell_{2}$, we define the unilateral shift operator $Z$ by

$$
\begin{gathered}
Z: \ell_{2}\left(\mathbb{R}^{n_{1}}, \mathbb{R}^{n_{2}}, \ldots\right) \rightarrow \ell_{2}\left(\mathbb{R}^{n_{0}}, \mathbb{R}^{n_{1}}, \mathbb{R}^{n_{2}}, \ldots\right) \\
\left(a_{1}, a_{2}, \ldots\right) \stackrel{Z}{\longmapsto}\left(0, a_{1}, a_{2}, \ldots\right) .
\end{gathered}
$$

Hence, $Z$ has the "infinite matrix" representation

$$
Z=\left[\begin{array}{llll}
0 & & & \\
I & 0 & & \\
0 & I & 0 & \\
& & & \ddots
\end{array}\right]
$$


where the dimensions of the zero and identity matrices are determined from the context. The adjoint of $Z$ is then defined by $Z^{*} x=\left(x_{1}, x_{2}, \ldots\right)$. We say a bounded linear operator $Q$ mapping $\ell_{2}\left(\mathbb{R}^{m_{0}}, \mathbb{R}^{m_{1}}, \ldots\right)$ to $\ell_{2}\left(\mathbb{R}^{n} 0, \mathbb{R}^{n_{1}}, \ldots\right)$ is block-diagonal if there exists a sequence of matrices $Q_{k}$ in $\mathbb{R}^{n_{k} \times m_{k}}$ such that, for all $w$, $z$, if $z=Q w$, then $z_{k}=Q_{k} w_{k}$. Then $Q$ has the representation $\operatorname{diag}\left(Q_{0}, Q_{1}, Q_{2}, \ldots\right)$. Observe that

$$
\begin{aligned}
& Z^{*} Q Z=\operatorname{diag}\left(Q_{1}, Q_{2}, \ldots\right) \text { and } \\
& Z Q Z^{*}=\operatorname{diag}\left(0, Q_{0}, Q_{1}, \ldots\right) .
\end{aligned}
$$

\section{PROBlem Formulation}

We start by giving a precise definition of an eventually periodic operator.

Definition 1: An operator $P$ on $\ell_{2}$ is $(h, q)$-eventually periodic if, for some integers $h \geq 0, q \geq 1$, we have

$$
Z^{q}\left(\left(Z^{*}\right)^{h} P Z^{h}\right)=\left(\left(Z^{*}\right)^{h} P Z^{h}\right) Z^{q}
$$

Furthermore, $P$ is called $h$-eventually time-invariant when $q=1$ and $q$-periodic when $h=0$.

So, a block-diagonal operator $Q$ is $(h, q)$-eventually periodic when $Q$ has the representation $\operatorname{diag}\left(Q_{0}, Q_{1}, \ldots, Q_{h-1}, \hat{Q}, \hat{Q}, \ldots\right)$, where $\hat{Q}=\operatorname{diag}\left(Q_{h}, Q_{h+1}, \ldots, Q_{h+q-1}\right)$.

Let $G$ be an LTV discrete-time system defined by the following statespace equation:

$$
\left[\begin{array}{c}
x_{k+1} \\
z_{k} \\
y_{k}
\end{array}\right]=\left[\begin{array}{ccc}
A_{k} & B_{1 k} & B_{2 k} \\
C_{1 k} & D_{11 k} & D_{12 k} \\
C_{2 k} & D_{21 k} & 0
\end{array}\right]\left[\begin{array}{c}
x_{k} \\
w_{k} \\
u_{k}
\end{array}\right] \quad x_{0} \neq 0
$$

for $w \in \ell_{2}$, where the sequences of these real state-space matrices define $(h, q)$-eventually periodic block-diagonal operators $A, B_{i}, C_{i}$, and $D_{i j}$ for $i, j=1,2$; thus $A$, for instance, is of the form $A=\operatorname{diag}\left(A_{0}, A_{1}, \ldots, A_{h-1}, \hat{A}, \hat{A}, \ldots\right)$, where $\hat{A}=\operatorname{diag}\left(A_{h}, A_{h+1}, \ldots, A_{h+q-1}\right)$. The signals $w_{k}$ and $z_{k}$ denote the exogenous disturbances and errors, respectively, whereas $u_{k}$ denotes the applied control and $y_{k}$ the measurements. The vectors $x_{k}$, $z_{k}, w_{k}, y_{k}$, and $u_{k}$ are real and have time-varying dimensions which we denote by $n_{k}, n_{z k}, n_{w k}, n_{y k}$, and $n_{u k}$ respectively. Notice that each matrix $A_{k}$ here has dimension $n_{k+1} \times n_{k}$.

We will say this system is stable when $I-Z A$ has a bounded inverse; this is equivalent to exponential stability as shown in [14]. Since plant $G$ is eventually periodic in our case, then its stability boils down to the stability of its periodic part, and thus, $G$ is (exponentially) stable if and only if the spectral radius of the matrix given below is strictly less than one (see [21]):

$$
\left[\begin{array}{cc}
0 & A_{h+q-1} \\
A_{L} & 0
\end{array}\right] \text {, with } A_{L}=\operatorname{diag}\left(A_{h}, A_{h+1}, \ldots, A_{h+q-2}\right) \text {. }
$$

The following definition expresses our synthesis goal.

Definition 2: A discrete-time LTV feedback controller $K$, with zero initial state and possibly time-varying dimensions, is a $\gamma$-admissible synthesis for plant $G$ if the closed-loop system in Fig. 1 is stable and the performance inequality (1) is achieved.

The operator theoretic machinery and results of [1], [15] are developed for LTV systems with zero initial conditions. As these refer-

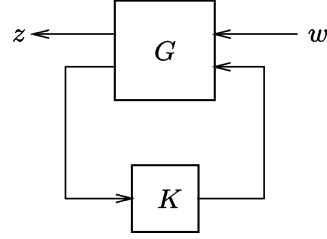

Fig. 1. Closed-loop system.

ences constitute the basis for this work, it is hence convenient to construct from $G$ an isomorphic $(h+1, q)$-eventually periodic system $\bar{G}$ having a zero initial condition. The uncertain initial condition $x_{0}$ from $G$ will be lumped with the disturbance $w$ in this isomorphic system. The state-space operators of $\bar{G}$, which we denote by block-diagonal operators $\bar{A}, \bar{B}_{1}, \bar{B}_{2}$ and so on, are defined as follows: $\bar{A}=Z A Z^{*}$, $\bar{B}_{2}=Z B_{2} Z^{*}, \bar{C}_{i}=Z C_{i} Z^{*}, \bar{D}_{i j}=Z D_{i j} Z^{*}$ for $i, j=1,2$; $Z^{*} \bar{B}_{1} Z=B_{1}$ and $\bar{B}_{10}=I_{n_{0}}$. The system $\bar{G}$ may be written more compactly in operator form as

$$
\left[\begin{array}{c}
\bar{x} \\
\bar{z} \\
\bar{y}
\end{array}\right]=\left[\begin{array}{ccc}
Z \bar{A} & Z \bar{B}_{1} & Z \bar{B}_{2} \\
\bar{C}_{1} & \bar{D}_{11} & \bar{D}_{12} \\
\bar{C}_{2} & \bar{D}_{21} & 0
\end{array}\right]\left[\begin{array}{c}
\bar{x} \\
\bar{w} \\
\bar{u}
\end{array}\right]
$$

where $\bar{w}_{0}=x_{0}, Z^{*} \bar{w}=w$, and $\bar{r}=Z r$ for $r=x, z, u, y$. We may then rephrase the synthesis objective as follows: a feedback controller $\bar{K}$ is a $\gamma$-admissible synthesis for $\bar{G}$ if the closed-loop system, denoted by $\bar{M}$, is stable and further the square $\ell_{2}$ induced norm of $\bar{M}$, defined by

$$
\|\bar{M}\|_{s q}:=\sup _{\left\|x_{0}\right\| \leq 1,\|w\| \leq 1}\|\bar{M} \bar{w}\|,
$$

satisfies the performance condition $\|\bar{M}\|_{s q}<\gamma$. Notice that if $\left(\bar{A}^{K}, \bar{B}^{K}, \bar{C}^{K}, \bar{D}^{K}\right)$ is the system realization for $\bar{K}$, then the corresponding realization for controller $K$ from Definition 2 is $\left(Z^{*} \bar{A}^{K} Z, Z^{*} \bar{B}^{K} Z, Z^{*} \bar{C}^{K} Z, Z^{*} \bar{D}^{K} Z\right)$.

\section{Main Results}

We start this section with an important theorem which follows from the results of [15].

Theorem 3: The closed-loop performance inequality $\|\bar{M}\|_{s q}<\gamma$ holds for some $\gamma$ if and only if there exist positive scalars $e, f_{1}$, and $f_{2}$, and associated block-diagonal operators

$$
\begin{aligned}
& E:=\operatorname{diag}(e I, e I, e I, \ldots) \text { and } \\
& F:=\operatorname{diag}\left(f_{1} I, f_{2} I, f_{2} I, \ldots\right)
\end{aligned}
$$

such that $e+f_{1}+f_{2}<2 \gamma$ and $\left\|E^{-1 / 2} \bar{M} F^{-1 / 2}\right\|_{\ell_{2} \rightarrow \ell_{2}}<1$.

Proof: Although the results of [15] are developed for LTV systems with constant dimensions and constant number of input and output channels, these results can be immediately extended to the case of nonstationary dimensions and time-varying number of channels. One easy way to see this is by augmenting the state-space matrices of the LTV system with zero blocks to accommodate for the time-varying dimensions and number of channels. With this said, the isomorphic system $\bar{G}$ can be equivalently reformulated into an LTV system with two exogenous input channels and one exogenous output channel. Specifically, in addition to the disturbance input channel, we will have an- 
other input channel associated with the uncertain initial state. This additional channel is only relevant at time $k=0$, where it has a value of $x_{0}$. As for the other time instants, the values of this channel are irrelevant as they would be annulled by the corresponding zero blocks in the state-space matrices. On the other hand, the disturbance input channel is only relevant at time instants $k>0$. Then, the "if" direction follows immediately from [15, Theorem 3.4],. Since the product of the number of exogenous input channels times the number of exogenous output channels is 2 , then invoking a version of [15, Theorem3.5], for sequences in real spaces, as suggested by [15, Remark 3.6], proves the "only if" direction.

Before stating the next result, we need to make a couple of definitions. Suppose $Q$ is an $(h, q)$-eventually periodic block-diagonal operator, then we define $\tilde{Q}$ to be the finite-horizon-first-period truncation of $Q$, namely $\tilde{Q}:=\operatorname{diag}\left(Q_{0}, Q_{1}, \ldots, Q_{h+q-1}\right)$. We also define

$$
\Xi(\tilde{Q}):=\operatorname{diag}\left(Q_{1}, \ldots, Q_{h+q-1}, Q_{h}\right)
$$

and the set

$\tilde{\mathcal{X}}=\left\{\tilde{X} \mid \tilde{X}=\operatorname{diag}\left(X_{0}, X_{1}, \ldots, X_{h+q-1}\right) \succ 0\right.$ and $\left.X_{i} \in \mathbb{R}^{n_{i} \times n_{i}}\right\}$.

We now give the main result of this note.

Theorem 4: Suppose that $G$ is an $(h, q)$-eventually periodic system with an uncertain initial condition $x_{0}$. Then there exists a $\gamma$-admissible $(h, q)$-eventually periodic synthesis $K$ for $G$ for some $\gamma$ if and only if there exist positive scalars $e, f_{1}, f_{2}, p, t$, and matrices $\tilde{R}, \tilde{S} \in \tilde{\mathcal{X}}$ such that

$$
\begin{aligned}
& e+f_{1}+f_{2}<2 \gamma, \quad S_{0} \prec f_{1} I, \\
& N_{R}^{*}\left\{L\left[\begin{array}{cc}
\tilde{R} & 0 \\
0 & p I
\end{array}\right] L^{*}-\left[\begin{array}{cc}
\Xi(\tilde{R}) & 0 \\
0 & e I
\end{array}\right]\right\} N_{R} \prec 0 \\
& N_{S}^{*}\left\{L^{*}\left[\begin{array}{cc}
\Xi(\tilde{S}) & 0 \\
0 & t I
\end{array}\right] L-\left[\begin{array}{cc}
\tilde{S} & 0 \\
0 & f_{2} I
\end{array}\right]\right\} N_{S} \prec 0, \\
& {\left[\begin{array}{cc}
\tilde{R} & I \\
I & \tilde{S}
\end{array}\right] \succeq 0, \quad\left[\begin{array}{cc}
p & 1 \\
1 & f_{2}
\end{array}\right] \succeq 0, \quad\left[\begin{array}{ll}
t & 1 \\
1 & e
\end{array}\right] \succeq 0}
\end{aligned}
$$

where

$$
L=\left[\begin{array}{cc}
\tilde{A} & \tilde{B}_{1} \\
\tilde{C}_{1} & \tilde{D}_{11}
\end{array}\right]
$$

and

$$
\begin{array}{ll}
\operatorname{Im} N_{R}=\operatorname{ker}\left[\begin{array}{ll}
\tilde{B}_{2}^{*} & \tilde{D}_{12}^{*}
\end{array}\right], & N_{R}^{*} N_{R}=I, \\
\operatorname{Im} N_{S}=\operatorname{ker}\left[\begin{array}{ll}
\tilde{C}_{2} & \tilde{D}_{21}
\end{array}\right], & N_{S}^{*} N_{S}=I .
\end{array}
$$

Proof: To start, consider the $(h+1, q)$-eventually periodic system $\bar{G}$ defined in (3). This system is isomorphic to plant $G$, and as a result, the existence of a $\gamma$-admissible $(h, q)$-eventually periodic synthesis $K$ for $G$ is equivalent to the existence of a $\gamma$-admissible $(h+1, q)$-eventually periodic synthesis $\bar{K}$ for $\bar{G}$. By Theorem 3, this is also equivalent to saying that $\bar{K}$ stabilizes the closed-loop system $\bar{M}$ and further achieves the inequality $\left\|E^{-1 / 2} \bar{M} F^{-1 / 2}\right\|_{\ell_{2} \rightarrow \ell_{2}}<1$ for some $E$ and $F$, defined as in (4) with positive scalars $e, f_{1}$, and $f_{2}$ satisfying $e+f_{1}+f_{2}<2 \gamma$. In other words, a solution $K$ exists if and only if a 1-admissible (or, simply, admissible) synthesis $\bar{K}$ exists for the scaled $(h+1, q)$-eventually periodic system

$$
\begin{aligned}
\bar{G}^{s} & =\left[\begin{array}{c|cc}
\bar{A}^{s} & \bar{B}_{1}^{s} & \bar{B}_{2}^{s} \\
\hline \bar{C}_{1}^{s} & \bar{D}_{11}^{s} & \bar{D}_{12}^{s} \\
\bar{C}_{2}^{s} & \bar{D}_{21}^{s} & 0
\end{array}\right] \\
& =\left[\begin{array}{c|cc}
\bar{A} & \bar{B}_{1} F^{-1 / 2} & \bar{B}_{2} \\
\hline E^{-1 / 2} \bar{C}_{1} & E^{-1 / 2} \bar{D}_{11} F^{-1 / 2} & E^{-1 / 2} \bar{D}_{12} \\
\bar{C}_{2} & \bar{D}_{21} F^{-1 / 2} & 0
\end{array}\right],
\end{aligned}
$$

for some $E$ and $F$. Since the system $\bar{G}^{s}$ has a zero initial condition, then by [1, Theorem 15], $\bar{K}$ exists if and only if, for all $k=0,1, \ldots, h+q$, there exist matrices $X_{k} \succ 0, Y_{k} \succ 0$, and $X_{h+q+1}=X_{h+1}, Y_{h+q+1}=Y_{h+1}$ such that

$$
\begin{array}{r}
N_{X k}^{*}\left\{\bar{L}_{k}^{s}\left[\begin{array}{cc}
X_{k} & 0 \\
0 & I
\end{array}\right]\left(\bar{L}_{k}^{s}\right)^{*}-\left[\begin{array}{cc}
X_{k+1} & 0 \\
0 & I
\end{array}\right]\right\} N_{X k} \prec 0 \\
N_{Y k}^{*}\left\{\left(\bar{L}_{k}^{s}\right)^{*}\left[\begin{array}{cc}
Y_{k+1} & 0 \\
0 & I
\end{array}\right] \bar{L}_{k}^{s}-\left[\begin{array}{cc}
Y_{k} & 0 \\
0 & I
\end{array}\right]\right\} N_{Y k} \prec 0 \\
{\left[\begin{array}{cc}
X_{k} & I \\
I & Y_{k}
\end{array}\right] \succeq 0}
\end{array}
$$

where $\operatorname{Im} N_{X \underline{k}}=\operatorname{ker}\left[\begin{array}{ll}\bar{B}_{2 k}^{s *} & \bar{D}_{12 k}^{s *}\end{array}\right], \operatorname{Im} N_{Y k}=\operatorname{ker}\left[\begin{array}{ll}\bar{C}_{2 k}^{s} & \bar{D}_{21 k}^{s}\end{array}\right]$, and $\bar{L}_{k}^{s}=\left[\begin{array}{cc}\bar{A}_{k}^{s} & \bar{B}_{1 k}^{s} \\ \bar{C}_{1 k}^{s} & \bar{D}_{11 k}^{s}\end{array}\right]$.

(Only if): Given an admissible synthesis $\bar{K}$, and hence inequalities (11)-(13) hold for $k=0,1, \ldots, h+q$, we now show that this implies the validity of the synthesis conditions (5)-(8). To begin, it is not difficult to see that inequalities (11) and (12), evaluated at time $k=0$, reduce respectively to the following conditions: (i) $X_{1} \succ 1 / f_{1} I$; and (ii) $Y_{1} \prec f_{1} I$. Applying the Schur complement formula to the coupling condition (13), we get $X_{1} \succeq Y_{1}^{-1}$; this, together with $Y_{1}^{-1} \succ 1 / f_{1} I$ from (ii), gives condition (i). Thus, $X_{1} \succ 1 / f_{1} I$ is a redundant condition, and so at time $k=0$, inequalities (11) and (12) reduce to the condition $S_{0} \prec f_{1} I$ in (5), where $S_{0}=Y_{1}$. We now focus on inequality (11) for $k=1, \ldots, h+q$. Setting $\tilde{R}=\operatorname{diag}\left(X_{1}, \ldots, X_{h+q}\right)$, then from the definitions in (3) and (10), we can equivalently write (11) for $k=1, \ldots, h+q$ as

$$
W^{*}\left\{V\left[\begin{array}{cc}
\tilde{R} & 0 \\
0 & I
\end{array}\right] V^{*}-\left[\begin{array}{cc}
\Xi(\tilde{R}) & 0 \\
0 & I
\end{array}\right]\right\} W \prec 0
$$

where

$$
W=\left(\left[\begin{array}{cc}
I & 0 \\
0 & e^{1 / 2} I
\end{array}\right] N_{R}\right)
$$

and

$$
V=\left[\begin{array}{cc}
\tilde{A} & f_{2}^{-1 / 2} \tilde{B}_{1} \\
e^{-1 / 2} \tilde{C}_{1} & \left(e f_{2}\right)^{-1 / 2} \tilde{D}_{11}
\end{array}\right]
$$

Some algebra leads to

$$
N_{R}^{*}\left\{L\left[\begin{array}{cc}
\tilde{R} & 0 \\
0 & \frac{1}{f_{2}} I
\end{array}\right] L^{*}-\left[\begin{array}{cc}
\Xi(\tilde{R}) & 0 \\
0 & e I
\end{array}\right]\right\} N_{R} \prec 0
$$


where $L$ is defined in (9). Similarly, setting $\tilde{S}=\operatorname{diag}\left(Y_{1}, \ldots, Y_{h+q}\right)$, we can equivalently write (12) for $k=1, \ldots, h+q$ as

$$
N_{S}^{*}\left\{L^{*}\left[\begin{array}{cc}
\Xi(\tilde{S}) & 0 \\
0 & \frac{1}{e} I
\end{array}\right] L-\left[\begin{array}{cc}
\tilde{S} & 0 \\
0 & f_{2} I
\end{array}\right]\right\} N_{S} \prec 0 .
$$

Last, setting $p=1 / f_{2}$ and $t=1 / e$, and noticing that these equalities satisfy the pertinent coupling conditions in (8), complete the proof of the "only if" direction.

(If): Given that the synthesis conditions (5)-(8) hold, we need to show that a $\gamma$-admissible synthesis $K$ for plant $G$ exists. Applying the Schur complement formula to the second coupling condition in (8), we get $p \geq 1 / f_{2}$, and hence we can write

$$
\left[\begin{array}{cc}
\tilde{R} & 0 \\
0 & \frac{1}{f_{2}} I
\end{array}\right] \preceq\left[\begin{array}{cc}
\tilde{R} & 0 \\
0 & p I
\end{array}\right],
$$

and as a result, we have

$$
\begin{aligned}
& N_{R}^{*}\left\{L\left[\begin{array}{cc}
\tilde{R} & 0 \\
0 & \frac{1}{f_{2}} I
\end{array}\right] L^{*}-\left[\begin{array}{cc}
\Xi(\tilde{R}) & 0 \\
0 & e I
\end{array}\right]\right\} N_{R} \\
& \preceq N_{R}^{*}\left\{L\left[\begin{array}{cc}
\tilde{R} & 0 \\
0 & p I
\end{array}\right] L^{*}-\left[\begin{array}{cc}
\Xi(\tilde{R}) & 0 \\
0 & e I
\end{array}\right]\right\} N_{R} \\
& \quad \prec 0 .
\end{aligned}
$$

Similarly, applying the Schur complement formula to the third coupling condition in (8), we get $t \geq 1 / e$, and consequently, we have

$$
N_{S}^{*}\left\{L^{*}\left[\begin{array}{cc}
\Xi(\tilde{S}) & 0 \\
0 & \frac{1}{e} I
\end{array}\right] L-\left[\begin{array}{cc}
\tilde{S} & 0 \\
0 & f_{2} I
\end{array}\right]\right\} N_{S} \prec 0 .
$$

Applying the reverse argument to the one used in the "only if" proof, we find that the preceding inequalities, together with $S_{0} \prec f_{1} I$ and the first coupling condition in (8), are equivalent to inequalities (11)-(13), where $X_{k+1}=R_{k}$ and $Y_{k+1}=S_{k}$ for $k=0,1, \ldots, h+q$; note that $X_{0}$ and $Y_{0}$ are inconsequential here, and are only required to satisfy the coupling condition (13), which is always possible. Thus, a $\gamma$-admissible synthesis $K$ exists as argued at the beginning of this proof.

Solutions $\tilde{R}, \tilde{S}, e$, and $f_{2}$ can then be used to construct an $(h, q)$-eventually periodic controller (with a zero initial condition). To do so, first form the following $(h, q)$-eventually periodic system, which is basically $\bar{G}^{s}$ from (10) sans the inconsequential part corresponding to $k=0$ :

$$
\left[\begin{array}{c|cc}
\tilde{A} & f_{2}^{-1 / 2} \tilde{B}_{1} & \tilde{B}_{2} \\
\hline e^{-1 / 2} \tilde{C}_{1} & \left(e f_{2}\right)^{-1 / 2} \tilde{D}_{11} & e^{-1 / 2} \tilde{D}_{12} \\
\tilde{C}_{2} & f_{2}^{-1 / 2} \tilde{D}_{21} & 0
\end{array}\right]
$$

Then, using $\tilde{R}$ and $\tilde{S}$, follow the procedure outlined in [1], [14] to construct an $(h, q)$-eventually periodic controller for this system.

Remark 5: From the preceding, it is clear that the initial condition $x_{0}$ is implicitly regarded here as a separate input channel of a similar nature to disturbance. With this in mind, it is sometimes convenient, as demonstrated in the concluding example, to redefine this input channel as $\hat{x}_{0}$ where $x_{0}=\Lambda \hat{x}_{0}$. In such a case, the performance inequality (1) would involve $\hat{x}_{0}$ instead of $x_{0}$, and the synthesis condition $S_{0} \prec f_{1} I$ in Theorem 4 should be replaced with $\Lambda^{*} S_{0} \Lambda \prec f_{1} I$.

Like in the case of zero initial states, if the synthesis conditions in Theorem 4 are invalid, we can only say that there exists no $\gamma$-admissible $(h, q)$-eventually periodic synthesis; but this does not necessarily imply the nonexistence of a different $\gamma$-admissible synthesis. Using a

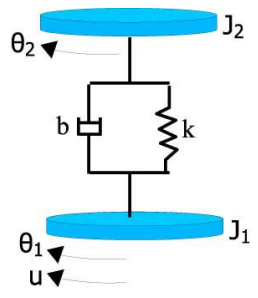

Fig. 2. Two-mass rotational system.

similar argument to that of the proof of [1, Lemma 7], it can be shown that, when it comes to eventually periodic plants, there exists a $\gamma$-admissible LTV synthesis if and only if there exists an eventually periodic one, having the same periodicity as the plant but probably a longer finite horizon. Now as an $(h, q)$-eventually periodic plant $G$ is also $(N, q)$-eventually periodic for all integers $N \geq h$, then if no $\gamma$-admissible $(h, q)$-eventually periodic synthesis for $G$ exists, we may still utilize the synthesis conditions of Theorem 4 as part of an algorithm to find, if possible, an $(M, q)$-eventually periodic controller for some $M>h$. Alternatively, given a stabilizable and detectable $(h, q)$-eventually periodic plant $G$ (which basically means that the periodic portion of $G$ is stabilizable and detectable), consider an increasing integer sequence of finite horizon lengths $N_{i}$ for $i=0,1,2, \ldots$, where $N_{0} \geq h$. Then, as $G$ is $\left(N_{i}, q\right)$-eventually periodic, it is always possible to use Theorem 4 to solve for a $\gamma_{i}$-admissible $\left(N_{i}, q\right)$-eventually periodic synthesis, where $\gamma_{i}$ denotes the minimum $\gamma$, up to a certain tolerance, that is achievable by an $\left(N_{i}, q\right)$-eventually periodic controller. Namely, the aforesaid synthesis is obtained by solving the convex optimization problem: minimize $\gamma$ subject to synthesis conditions (5)-(8) reformulated for an $\left(N_{i}, q\right)$-eventually periodic plant. It is obvious that solving this optimization problem for all $N_{i}$ would result in a non-increasing sequence of optimal values $\gamma_{i}$, which could be used as a guideline for choosing a synthesis of reasonable size and performance.

Clearly, an LTI system is an $(h, q)$-eventually periodic system with $h=0$ and $q=1$. With this said, the next result follows immediately from Theorem 4.

Corollary 6: Suppose that $G$ is an LTI system with an uncertain initial condition, and state-space matrices $A, B_{i}, C_{i}, D_{i j}$ for $i, j=1$, 2, with $D_{22}=0$. Then, given a non-negative integer $N$, there exists a $\gamma$-admissible $N$-eventually time-invariant synthesis $K$ for some $\gamma$ if and only if, for all $k=0,1, \ldots, N$, there exist positive scalars $e, f_{1}$, $f_{2}, p, t$, matrices $R_{k} \succ 0, S_{k} \succ 0$, and $R_{N+1}=R_{N}, S_{N+1}=S_{N}$, such that

$$
\begin{aligned}
& e+f_{1}+f_{2}<2 \gamma, \quad S_{0} \prec f_{1} I, \\
& N_{R}^{*}\left\{L\left[\begin{array}{cc}
R_{k} & 0 \\
0 & p I
\end{array}\right] L^{*}-\left[\begin{array}{cc}
R_{k+1} & 0 \\
0 & e I
\end{array}\right]\right\} N_{R} \prec 0, \\
& N_{S}^{*}\left\{L^{*}\left[\begin{array}{cc}
S_{k+1} & 0 \\
0 & t I
\end{array}\right] L-\left[\begin{array}{cc}
S_{k} & 0 \\
0 & f_{2} I
\end{array}\right]\right\} N_{S} \prec 0, \\
& {\left[\begin{array}{cc}
R_{k} & I \\
I & S_{k}
\end{array}\right] \succeq 0, \quad\left[\begin{array}{cc}
p & 1 \\
1 & f_{2}
\end{array}\right] \succeq 0, \quad\left[\begin{array}{ll}
t & 1 \\
1 & e
\end{array}\right] \succeq 0}
\end{aligned}
$$

where $L=\left[\begin{array}{cc}A & B_{1} \\ C_{1} & D_{11}\end{array}\right], \operatorname{Im} N_{R}=\operatorname{ker}\left[\begin{array}{ll}B_{2}^{*} & D_{12}^{*}\end{array}\right], N_{R}^{*} N_{R}=I$, $\operatorname{Im} N_{S}=\operatorname{ker}\left[\begin{array}{ll}C_{2} & D_{21}\end{array}\right]$, and $N_{S}^{*} N_{S}=I$.

Note that an LTI system with an uncertain initial condition is isomorphic to a 1-eventually time-invariant system having a zero initial state. Hence, given such a system, it is very conceivable that an $N$-eventually time-invariant controller, for $N \geq 1$, would provide better performance than an LTI one. We give an example of such a system next. 

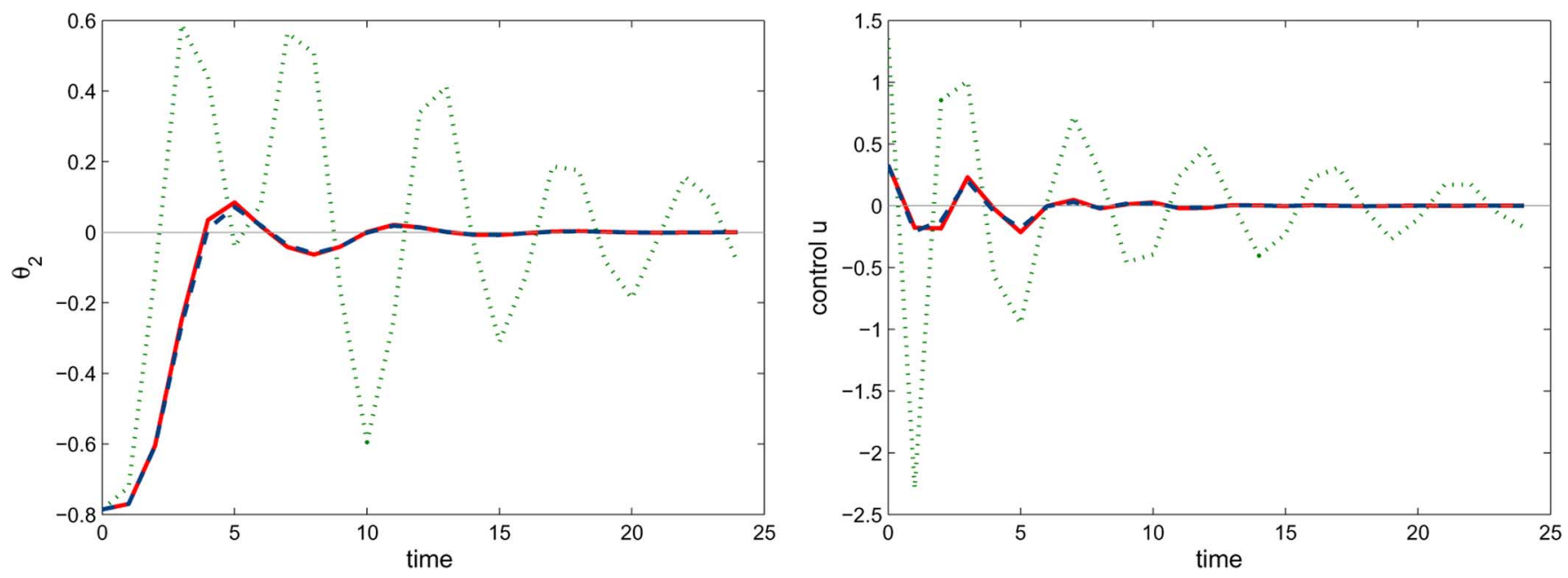

Fig. 3. LTI (dotted curve) versus 1-eventually time-invariant (solid curve) versus 1000-eventually time-invariant (dashed curve).

\section{ILLUSTRATIVE EXAMPLE}

Our primary goal in this section is to provide a simple example illustrating the approach of the paper. Consider the two-mass rotational system in Fig. 2. The state variables $\theta_{1}$ and $\theta_{2}$ denote the angular displacements of the bodies $J_{1}$ and $J_{2}$, respectively, with respect to some common reference. The system is in a stable equilibrium state when $\theta_{1}=\theta_{2}$ and the angular velocities $\dot{\theta}_{1}=\dot{\theta}_{2}=0$. The control input is the torque $u$ applied to the body $J_{1}$. Suppose that the inertias $J_{1}=1$, $J_{2}=0.1$, the spring constant $k=0.091$, and the damping coefficient $b=0.0036$. Given that the system is initially in an equilibrium state with the specific values of the angular displacements uncertain, then, using the synthesis approach herein, we would like to find a discrete-time controller, with a sampling period $T=1$, that would bring the system to the equilibrium position $\theta_{1}=\theta_{2}=0$. Suppose that the measurable output is only $\theta_{2}$. Then, setting $x=\left(\theta_{2}, \dot{\theta}_{2}, \theta_{1}, \dot{\theta}_{1}\right)$, we can write the following continuous-time state space equations:

$$
\begin{aligned}
& \dot{x}=\underbrace{\left[\begin{array}{cccc}
0 & 1 & 0 & 0 \\
-\frac{k}{J_{2}} & -\frac{b}{J_{2}} & \frac{k}{J_{2}} & \frac{b}{J_{2}} \\
0 & 0 & 0 & 1 \\
\frac{k}{J_{1}} & \frac{b}{J_{1}} & -\frac{k}{J_{1}} & -\frac{b}{J_{1}}
\end{array}\right]}_{A_{c}} x+\underbrace{\left[\begin{array}{c}
0 \\
0 \\
0 \\
\frac{1}{J_{1}}
\end{array}\right]}_{B_{c}} u, \\
& y=C_{c} x=\left[\begin{array}{llll}
1 & 0 & 0 & 0
\end{array}\right] x .
\end{aligned}
$$

The corresponding discrete-time model obtained by zero-order hold sampling is given by

$$
x_{k+1}=A x_{k}+B_{2} u_{k},
$$

where $x_{k}=x(k T), T=1$ is the sampling period, $A=\exp \left(A_{c} T\right)$ and

$$
B_{2}=\left(\int_{0}^{T} \exp \left(A_{c}(T-\tau)\right) d \tau\right) B_{c} .
$$

We assume that the disturbances are in the form of control inaccuracies $\delta u$ which do not exceed $10 \%$ of the desired control torque at each time instant, i.e., $\left|\delta u_{k}\right| \leq 0.1\left|u_{k}\right|$ for all $k \geq 0$. As for the exogenous errors to be controlled, we choose to equally penalize $\theta_{2}$ and $u$. Setting $w_{k}=\delta u_{k}$, the preceding leads to the following discrete-time LTI model:

$$
\left[\begin{array}{c}
x_{k+1} \\
z_{k} \\
y_{k}
\end{array}\right]=\left[\begin{array}{ccc}
A & B_{1} & B_{2} \\
C_{1} & 0 & D_{12} \\
C_{2} & 0 & 0
\end{array}\right]\left[\begin{array}{l}
x_{k} \\
w_{k} \\
u_{k}
\end{array}\right]
$$

where $B_{1}=B_{2}, C_{2}=C_{c}, C_{1}=\left[\begin{array}{llll}1 & 0 & 0 & 0 \\ 0 & 0 & 0 & 0\end{array}\right]$, and $D_{12}=\left[\begin{array}{l}0 \\ 1\end{array}\right]$.

Appealing to Remark 5, we define $\hat{x}_{0}=\theta_{2}(0)$, and hence $x_{0}=\Lambda \hat{x}_{0}$ with $\Lambda=\left[\begin{array}{llll}1 & 0 & 1 & 0\end{array}\right]^{*}$. Then, using SeDuMi [22] along with the interface [23], we solve the convex optimization problem: minimize $\gamma$ subject to linear matrix inequalities (LMIs) (14) for $N=0,1,1000$, where the condition $S \prec f_{1} I$ is replaced with $\Lambda^{*} S \Lambda \prec f_{1} I$. We obtain the optimal values $\gamma_{\text {opt }} \approx 10.31,4.65,4.65$, respectively. Notice that, for this example, the performance is significantly improved if we employ a 1-eventually time-invariant controller instead of an LTI one, whereas no notable change occurs when the finite-horizon length of the controller is made much larger than one. This is clearly shown in Fig. 3 which gives the simulation results for the case where $\theta_{2}(0)=-\pi / 4$ and $\delta u_{k}=0.1 u_{k}$ for all $k$. All computations are carried out in Matlab 7.1, using SeDuMi 1.1R3, on a Dell XPS laptop with Intel Core Duo 2 $\mathrm{GHz}$ processor and $1 \mathrm{~GB}$ of RAM running Windows XP. The elapsed (i.e., wall clock) times for solving these optimization problems are $0.2 \mathrm{sec}, 0.6 \mathrm{sec}$, and $14.5 \mathrm{~min}$ for $N=0,1,1000$ respectively.

\section{CONCLUSION}

This note solves the $\ell_{2}$-induced control problem for eventually periodic systems with uncertain initial states. Specifically, we derive exact conditions for the existence of a synthesis of the same eventually periodic class as the plant. This synthesis stabilizes the closed-loop system and further achieves a performance condition, in which the norm constraints placed on the uncertain initial state and the input disturbance are independent. A version of the main result is given for the special case of LTI systems, and an illustrative example is also provided.

\section{REFERENCES}

[1] M. Farhood and G. E. Dullerud, "LMI tools for eventually periodic systems," Syst. Control Lett., vol. 47, pp. 417-432, 2002. 
[2] M. Farhood and G. E. Dullerud, "On the $\ell_{2}$-induced control for eventually periodic systems," in Proc. Amer. Control Conf., 2004, pp. 2021-2026.

[3] M. Farhood and G. E. Dullerud, "Duality and eventually periodic systems," Int. J. Robust Nonlin. Control, vol. 15, no. 13, pp. 575-599, 2005.

[4] L. Vandenberghe and S. Boyd, "Semidefinite programming," SIAM Rev., vol. 38, no. 1, pp. 49-95, 1996.

[5] Y. Nesterov and M. J. Todd, "Self-scaled barriers and interior-point methods for convex programming," Math. Operat. Res., vol. 22, no. 1, pp. 1-42, 1997.

[6] S. Boyd and L. Vandenberghe, Convex Optimization. Cambridge, U.K.: Cambridge Univ. Press, 2004.

[7] M. S. Branicky, "Studies in Hybrid Systems: Modeling, Analysis, and Control," Ph.D. dissertation, Massachusetts Inst. Technol., Cambridge, 1995.

[8] D. Liberzon, Switching in Systems and Control. Boston, MA: Birkhäuser, 2003.

[9] E. Frazzoli, M. A. Dahleh, and E. Feron, "A hybrid control architecture for aggressive maneuvering of autonomous helicopters," in Proc. IEEE Conf. Decision Control, 1999, pp. 2471-2476.

[10] P. Gahinet and P. Apkarian, "A linear matrix inequality approach to $H_{\infty}$ control," Int. J. Robust Nonlin. Control, vol. 4, pp. 421-448, 1991.

[11] T. Iwasaki and R. E. Skelton, "All controllers for the general $H_{\infty}$ control problem: LMI existence conditions and state space formulas," $\mathrm{Au}$ tomatica, vol. 30, pp. 1307-1317, 1994.

[12] A. Packard, "Gain scheduling via linear fractional transformations," Syst. Control Lett., vol. 22, pp. 79-92, 1994.

[13] R. D'Andrea, "Generalized $\ell_{2}$ synthesis," IEEE Trans. Automat. Control, vol. 44, no. 6, pp. 1145-1156, Jun. 1999.

[14] G. E. Dullerud and S. G. Lall, "A new approach to analysis and synthesis of time-varying systems," IEEE Trans. Automat. Control, vol. 44, no. 8, pp. 1486-1497, Aug. 1999.

[15] C. L. Pirie and G. E. Dullerud, "Robust controller synthesis for uncertain time-varying systems," SIAM J. Control Optim., vol. 40, no. 4, pp. 1312-1331, 2002.

[16] M. Green and D. Limebeer, Linear Robust Control. Englewood Cliffs, NJ: Prentice Hall, 1995.

[17] I. Gohberg, M. A. Kaashoek, and L. Lerer, "Minimality and realization of discrete time-varying systems," Operator Theory: Adv. Appl., vol. 56, pp. 261-296, 1992.

[18] A. Varga, "Computation of minimal periodic realizations of transferfunction matrices," IEEE Trans. Automat. Control, vol. 49, no. 1, pp. 146-149, Jan. 2004.

[19] S. Lall and C. Beck, "Error-bounds for balanced model-reduction of linear time-varying systems," IEEE Trans. Automat. Control, vol. 48, no. 6, pp. 946-956, Jun. 2003.

[20] H. Sandberg and A. Rantzer, "Balanced truncation of linear time-varying systems," IEEE Trans. Automat. Control, vol. 49, no. 2, pp. 217-229, Feb. 2004.

[21] S. Lall, C. Beck, and G. E. Dullerud, "Guaranteed error bounds for model reduction of linear time-varying systems," in Proc. Amer. Control Conf., 1998, pp. 634-638.

[22] J. F. Sturm, "Using SeDuMi 1.02, a MATLAB toolbox for optimization over symmetric cones," Optim. Methods Software, vol. 11-12, pp. 625-653, 1999.

[23] Y. Labit, D. Peaucelle, and D. Henrion, "SEDUMI INTERFACE 1.02: A tool for solving LMI problems with SEDUMI," in Proc. IEEE Int. Symp. Computer Aided Control Syst. Design, Sep. 2002, pp. 272-277.

\section{Control Algorithms Along Relative Equilibria of Underactuated Lagrangian Systems on Lie Groups}

Nikolaj Nordkvist and Francesco Bullo, Senior Member, IEEE

\begin{abstract}
We present novel algorithms to control underactuated mechanical systems. For a class of invariant systems on Lie groups, we design iterative small-amplitude control forces to accelerate along, decelerate along, and stabilize relative equilibria. The technical approach is based upon a perturbation analysis and the design of inversion primitives and composition methods. We illustrate the algorithms on an underactuated planar rigid body and on a satellite with two thrusters.
\end{abstract}

Index Terms - Mechanical systems, nonlinear control, relative equilibria, underactuated systems.

\section{INTRODUCTION}

In this technical note we study control of underactuated mechanical systems on Lie groups. We focus on the particular class of motions called relative equilibria. A relative equilibrium is a motion for which the body-fixed velocity is constant while no control forces are applied; thus when referring to a relative equilibrium a specific body-fixed velocity is implied. Accelerating/decelerating along a relative equilibrium means increasing/decreasing the velocity in the direction of a relative equilibrium while the configuration behaves accordingly. We concentrate on the construction of small-amplitude control forces that, when used iteratively, result in a given acceleration/deceleration along a relative equilibrium; stabilization is achieved as zero acceleration. Perturbation analysis and Lie group theory play a crucial role in the analysis. Example systems to which the theory applies are a hovercraft, modeled as an underactuated planar rigid body, and a satellite with two thrusters.

The motivation for studying underactuated mechanical systems is twofold. First, control algorithms for underactuated systems enable more general control designs than those in fully actuated systems, e.g., less costly designs or lighter designs. Second, control algorithms for underactuated systems are applicable in the situation of an actuator failure and, therefore, they improve robustness of the control system; this robustness is crucial in case the vehicle is in a hazardous environment or is hardly accessible (e.g., a satellite).

A vast literature is available on mechanical control systems. Extensive research has focused on underactuated mechanical systems, especially in the context of controlled Lagrangians and Hamiltonians, e.g., see [1], [2] and subsequent works. Somehow less research is available for controlling systems along relative equilibria; a related spin-up problem is considered in [3], the theory of kinematic reductions is exposed in [4]. Since this document builds directly upon the work in [5] we refer the reader to that document for a literature survey relevant for control algorithms for underactuated Lagrangian systems on Lie groups. A generalization of the theory in [5] to a larger class of mechanical systems can be found in [6]. An advantage of our approach

Manuscript received January 26, 2007; revised January 10, 2008. Current version published December 10, 2008. This material is based upon work supported in part by the National Science Foundation Award CMS-0442041. Recommended by Associate Editor J. Berg.

N. Nordkvist is with the Department of Mathematics, Technical University of Denmark, Lyngby 2800 Kgs., Denmark (e-mail: n.nordkvist@gmail.com).

F. Bullo is with the Department of Mechanical Engineering, University of California, Santa Barbara, CA 93106 USA (e-mail: bullo@engineering.ucsb. edu).

Digital Object Identifier 10.1109/TAC.2008.2007143 